\title{
Supra-thermal viscosity of magnetized neutron star matter
}

\author{
Debarati Chatterjee \\ Institut für Theoretische Physik, University of Heidelberg, Germany \\ E-mail: D.ChatterjeedThPhys.Uni-Heidelberg.DE \\ Jürgen Schaffner-Bielich \\ Institut für Theoretische Physik, University of Heidelberg, Germany \\ E-mail: SchaffnerdThPhys.Uni-Heidelberg.DE
}

\begin{abstract}
The growth of unstable modes in neutron stars could result in copious emission of gravitational waves, which the upcoming GW detectors aim to detect. The gravitational waves from these unstable modes carry signatures of the composition of dense neutron star matter, and provide a unique tool to probe the stellar interior. The r-mode is one such mode, related to the rotation of the star, that grows rapidly in amplitude until it is saturated by non-linear effects. Bulk viscosity is one of the prime factors that determines whether r-modes become unstable. We study how magnetic field affects the bulk viscosity of dense hadronic matter due to the direct Urca process, incorporating non-linear effects in the supra-thermal regime.
\end{abstract}

25th Texas Symposium on Relativistic Astrophysics

December 6-10, 2010

Heidelberg, Germany 


\section{Introduction}

The composition of dense matter in the neutron star core is yet unknown. The internal structure of neutron stars can be probed through the study of unstable modes of oscillation in rotating neutron stars. Non-radial oscillation modes can become unstable, resulting in emission of gravitational waves. These gravitational waves carry the signature of neutron star matter. Several upcoming interferometric detectors aim to detect gravitational waves from such unstable modes. The r-mode is one such mode, restored by the Coriolis force, and is generic to all rotating neutron stars [W]. Whether an r-mode becomes unstable is determined by whether gravitational wave growth is balanced by (shear or bulk) viscosity of neutron star matter. Bulk viscosity is produced when compression or expansion of a fluid element due to r-mode oscillation leads to departure from chemical equilibrium [ [, [3]. Weak interaction processes among particles restore chemical equilibrium. There exists a window of instability where viscous processes cannot damp the r-mode instability [可]. The amplitude of unstable r-modes grows exponentially with time, till they are saturated by nonlinear effects [回]. It is interesting to study whether the nonlinear bulk viscosity could lead to the saturation of the r-mode amplitude [ [6-8]

Pulsars are neutron stars with strong magnetic fields $\sim 10^{12}-10^{14}$ G. Observations of Soft Gamma-ray Repeaters (SGRs) and Anomalous X-ray Pulsars (AXPs) suggest the existence of a class of neutron stars called "magnetars" with strong surface magnetic fields $\sim 10^{14}-10^{15} \mathrm{G}$. The value of the interior magnetic field is expected to be higher, accounting for the high conductivity of the core and the possible twisting of the magnetic field [9]. The scalar virial theorem allows interior fields as high as $10^{18} \mathrm{G}$. If superstrong magnetic fields exist in the interior, new particle reaction channels could open and standard processes would be modified, changing the evolution of r-modes. We study the effect of strong magnetic fields in neutron stars on nonlinear bulk viscosity and examine the effect it may have on the saturation of the growth of r-modes.

\section{Bulk viscosity: sub- and suprathermal regime}

In the neutron star core, where stellar matter consists of neutrons $n$, protons $p$, electrons $e$ and muons $\mu$, bulk viscosity is mainly produced by the direct Urca process,

$$
n \rightarrow p+l+\bar{v}_{l}, p+l \rightarrow n+v_{l}
$$

where $l$ stands for leptons $(e, \mu)$ and $v_{l}$ is the corresponding neutrino. The chemical potential imbalance $\delta \mu=\mu_{n}-\mu_{p}-\mu_{l}=0$ at $\beta$-equilibrium, when the forward and backward reaction rates are equal. An external perturbation due to the r-mode oscillation results in departure from chemical equilibrium and a difference between forward and backward reaction rates, proportional to the chemical potential imbalance. The net reaction rate per unit volume is

$$
\Gamma=\lambda \delta \mu,
$$

$\lambda$ being a coefficient to be determined later. The relaxation time $\tau$ is given by [四]

$$
\frac{1}{\tau}=\frac{\Gamma}{\delta \mu} \frac{\delta \mu}{n_{b} \delta x_{n}} .
$$


Here $\delta x_{n}=x_{n}-\bar{x}_{n}$ is the departure of the neutron fraction from its thermodynamic equilibrium value $\bar{x}_{n}$ in the unperturbed state. The general expression for the real part of bulk viscosity coefficient [四] is

$$
\operatorname{Re} \zeta=-\frac{n_{b}^{2} \tau}{1+(\omega \tau)^{2}} \frac{\partial P}{\partial n_{n}} \frac{d \bar{x}_{n}}{d n_{b}},
$$

where $\tau$ is determined from (ㄷ.3]) and the other terms can be determined from the equation of state. Here $\bar{x}_{n}=\frac{n_{n}}{n_{b}}$ gives the neutron fraction in the equilibrium state, $P$ is the pressure and $n_{b}=\sum_{B} n_{B}$ is the total baryon density. In the co-rotating frame, the generic equation relating the angular velocity $\omega$ of the $(l, m)$ r-mode to the angular velocity of rotation of the star $\Omega$ is $\omega=\frac{2 m}{l(l+1)} \Omega$ [四].

The net reaction rate (․2.) can be expressed as $\Gamma=\Gamma_{0} \Delta I$, in terms of a numerical prefactor $\Gamma_{0}$ [ए]]. Introducing the dimensionless quantities [ए]]

$$
x_{i}=\frac{\varepsilon_{i}-\mu_{i}}{T}, x_{v}=\frac{\varepsilon_{v}}{T}, \xi=\frac{\delta \mu}{T},
$$

where $\varepsilon_{i}$ are the energies of the particles, $\mu_{i}$ the chemical potentials and $T$ is the temperature, we can write

$$
\Delta I=\int_{0}^{\infty} d x_{v} x_{v}^{2}\left[J\left(x_{v}-\xi\right)-J\left(x_{v}+\xi\right)\right],
$$

with the function

$$
J(x)=\int d x_{n} d x_{p} d x_{l} f\left(x_{n}\right) f\left(x_{p}\right) f\left(x_{l}\right) \delta\left(x_{n}+x_{p}+x_{l}-x\right) .
$$

The function can be evaluated analytically as [ए]]:

$$
J(x)=\frac{x^{4}+10 \pi^{2} x^{2}+9 \pi^{4}}{24\left(e^{x}+1\right)}
$$

resulting in

$$
\begin{array}{r}
\Delta I=\frac{17 \pi^{4}}{60} \xi F(\xi), \\
F(\xi)=1+\frac{10 \xi^{2}}{17 \pi^{2}}+\frac{10 \xi^{4}}{17 \pi^{4}} .
\end{array}
$$

This yields the coefficient in (2.2) to be

$$
\lambda=\frac{\Gamma_{0}}{T} \frac{\Delta I}{\xi}
$$

In the subthermal regime, $\delta \mu<<T$, and hence $F(\xi)=1$. For the evaluation of $\lambda$ in the suprathermal regime $\delta \mu>>T$, we calculate the difference in chemical potential $\delta \mu$, induced by the external density perturbation $\Delta n_{b}$.

\subsection{Theoretical Model for the equation of state}

We adopt a relativistic field theoretical model to describe the $\beta$-equilibrated and charge neutral matter. The constituents are baryons, electrons and muons. In this model, the baryon-baryon 
interaction is mediated by the exchange of scalar $\sigma$, vector $\omega$ and isovector $\rho$ mesons. In the absence of a magnetic field, the Lagrangian density is given by [ए]]

$$
\begin{aligned}
\mathscr{L}_{B}= & \sum_{B} \bar{\Psi}_{B}\left(i \gamma_{\mu} \partial^{\mu}-m_{B}+g_{\sigma B} \sigma-g_{\omega B} \gamma_{\mu} \omega^{\mu}-g_{\rho B} \gamma_{\mu} \mathrm{t}_{B} \cdot \rho^{\mu}\right) \Psi_{B} \\
& +\frac{1}{2}\left(\partial_{\mu} \sigma \partial^{\mu} \sigma-m_{\sigma}^{2} \sigma^{2}\right)-U(\sigma) \\
& -\frac{1}{4} \omega_{\mu v} \omega^{\mu v}+\frac{1}{2} m_{\omega}^{2} \omega_{\mu} \omega^{\mu}-\frac{1}{4} \rho_{\mu \nu} \cdot \rho^{\mu v}+\frac{1}{2} m_{\rho}^{2} \rho_{\mu} \cdot \rho^{\mu} .
\end{aligned}
$$

The Dirac bispinor $\Psi_{B}$ represents the isospin multiplets for baryons B, having vacuum nucleon mass $m_{B}, \mathrm{t}_{B}$ is the isospin operator, and $\omega_{\mu v}$ and $\rho_{\mu v}$ are field strength tensors. The scalar selfinteraction term [12]

$$
U(\sigma)=\frac{1}{3} g_{2} \sigma^{3}+\frac{1}{4} g_{3} \sigma^{4}
$$

is introduced to obtain the correct compressibility of nuclear matter. The calculation is performed using the mean field approximation [피]. The scalar density and baryon number density are respectively

$$
\begin{gathered}
n_{B}^{S}=\frac{2 J_{B}+1}{2 \pi^{2}} \int_{0}^{k_{F_{B}}} \frac{m_{B}^{*}}{\left(k^{2}+m_{B}^{* 2}\right)^{1 / 2}} k^{2} d k, \\
n_{B}=\left(2 J_{B}+1\right) \frac{k_{F_{B}}^{3}}{6 \pi^{2}},
\end{gathered}
$$

where $k_{F_{B}}$ is the Fermi momentum, $J_{B}$ is the spin, and $I_{3 B}$ the isospin projection. The effective mass and the chemical potential of the baryon $B$ are $m_{B}^{*}=m_{B}-g_{\sigma B} \sigma$ and $\mu_{B}=\left(k_{F_{B}}^{2}+m_{B}^{* 2}\right)^{1 / 2}+$ $g_{\omega B} \omega_{0}+I_{3 B} g_{\rho B} \rho_{03}$, respectively.

\subsection{Effects from the magnetic field}

We now consider effects from a strong magnetic field on the properties of dense matter. The charged particles (electrons, protons) are quantized in Landau levels perpendicular to the direction of the magnetic field [14]. The number of Landau levels populated is sensitive to the magnetic field strength and the baryon density.

An important effect of the magnetic field is the opening of the direct Urca process in the regime $k_{F_{n}}>k_{F_{p}}+k_{F_{e}}$, which is otherwise forbidden by momentum conservation. For the direct Urca process in a magnetic field, the net reaction rate is [144, [5]]

$$
\Gamma_{l}=\frac{32 \pi G_{F}^{2} \cos ^{2} \theta_{c} m_{n}^{*} m_{p}^{*} \mu_{l}}{(2 \pi)^{5}} R_{B}^{q c} \int d \varepsilon_{v} \varepsilon_{v}^{2}\left\{J\left(\varepsilon_{v}-\delta \mu\right)-J\left(\varepsilon_{v}+\delta \mu\right)\right\}
$$

where the factor $R_{B}^{q c}$ as defined in [15] describes the effect of the magnetic field. Following the result of [प]],

$$
\int d \varepsilon_{v} \varepsilon_{v}^{2}\left\{J\left(\varepsilon_{v}-\delta \mu\right)-J\left(\varepsilon_{v}+\delta \mu\right)\right\}=\frac{17(\pi k T)^{4}}{60} \delta \mu F(\xi),
$$

from which we can calculate the suprathermal direct Urca bulk viscosity in a strong magnetic field. 


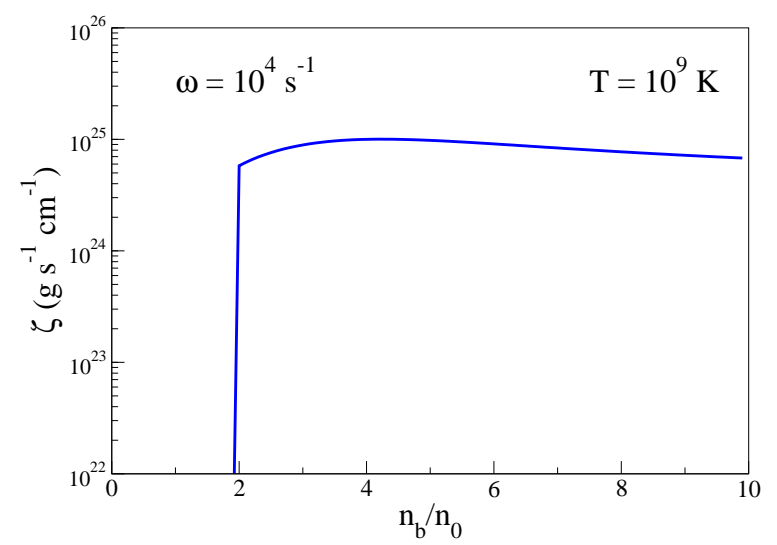

Figure 1: Subthermal bulk viscosity for $B=0$ at $T=10^{9} \mathrm{~K}$

\section{Results and Discussions}

We studied neutron star matter consisting of baryons (neutrons, protons, $\Lambda$ hyperons), electrons and muons. The nucleon-meson coupling constants are determined from the saturation properties of nuclear matter [ए]]. We adopt a binding energy of $E / A=-16.3 \mathrm{MeV}$, a baryon density of $n_{0}=0.153 \mathrm{fm}^{-3}$, an asymmetry energy coefficient of $a_{\text {asy }}=32.5 \mathrm{MeV}$, an incompressibility of $K=240 \mathrm{MeV}$, and an effective nucleon mass of $m_{N}^{*} / m_{N}=0.78$. The coupling of $\Lambda$ hyperons with $\omega$ mesons is determined from the $\mathrm{SU}(6)$ quark model, and the one for the $\sigma$ meson from a potential depth of $\Lambda$ hyperons in normal nuclear matter of $U_{\Lambda}=-30 \mathrm{MeV}$ [ㅁ] ]. We consider a constant and uniform magnetic field of $10^{17} \mathrm{G}$ for this calculation.

The coefficient of the bulk viscosity at $10^{9} \mathrm{~K}$ in the subthermal regime for zero magnetic field is plotted in Fig.1 as a function of the normalized baryon density. The bulk viscosity in the suprathermal regime in absence of the magnetic field at temperatures of $10^{6} \mathrm{~K}$ (Fig.2) and $10^{9} \mathrm{~K}$ (Fig.3) are plotted as a function of density perturbation amplitude at a baryon density $n_{b}=4 n_{0}$ and for $\omega=10^{4} \mathrm{~s}^{-1}$. As the r-mode enters the suprathermal regime, the amplitude rises steeply above the subthermal value by several orders of magnitude, eventually reaching a maximum, and decreases again. Near the maximum value, the large value of the suprathermal bulk viscosity can act as a more effective damping mechanism than in the subthermal case. In Fig.4, we compare the suprathermal bulk viscosities at $B=0$ and $B=10^{17} \mathrm{G}$ as a function of the perturbation amplitude. While the suprathermal bulk viscosity peaks near the perturbation amplitude of $\sim 0.1$ for $B=0$, it reaches its maximum much earlier $(\sim 0.01)$ in a $10^{17} \mathrm{G}$ field. This can result in an earlier saturation of the r-mode amplitude during its evolution. 


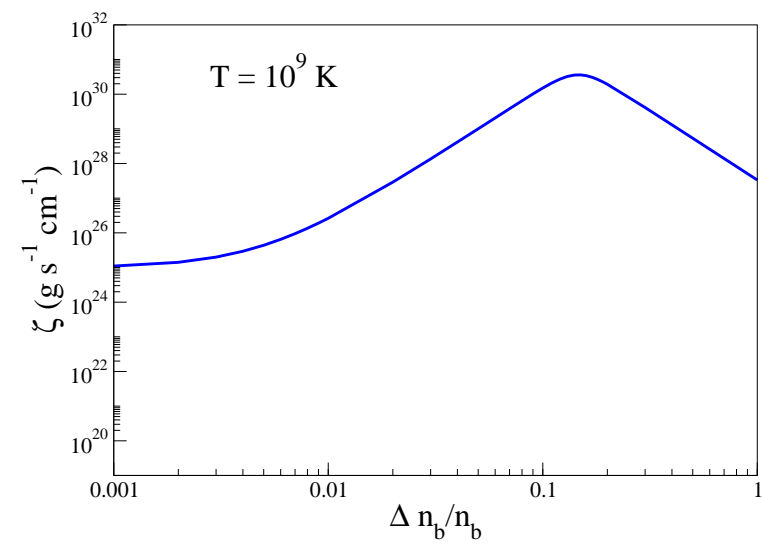

Figure 2: Suprathermal bulk viscosity for $B=0$ at $T=10^{9} \mathrm{~K}$

\section{Summary}

We studied the nonlinear behaviour of the direct Urca bulk viscosity at large amplitudes for magnetic fields of $B=0$ and $B=10^{17} \mathrm{G}$. The direct Urca bulk viscosity coefficient increases with the perturbation amplitude by several orders of magnitude in the suprathermal regime as it reaches a maximum. The large value of the suprathermal bulk viscosity near the maximum may be sufficient to saturate the growth of the r-mode amplitude. In the presence of a magnetic field, the suprathermal bulk viscosity saturates at a smaller oscillation amplitude compared to $B=0$, and could lead to a faster saturation of unstable r-modes. As r-modes regulate the spin frequency of newly born neutron stars, observations of neutron star rotation frequencies could be used to put constraints on the magnitude of magnetic fields in neutron stars.

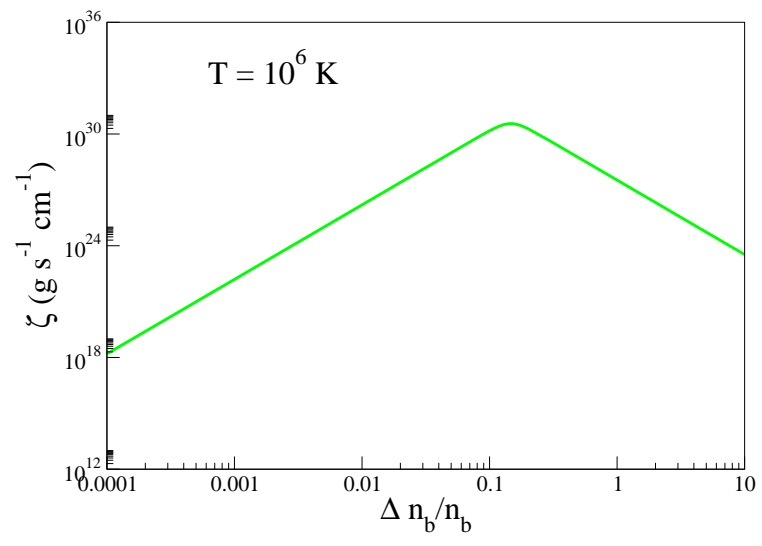

Figure 3: Suprathermal bulk viscosity for $B=0$ at $T=10^{6} \mathrm{~K}$

\section{References}

[1] N. Andersson, Class. Quant. Grav. 20, R105 (2003).

[2] P.B. Jones, Phys. Rev. Lett. 86, 1384 (2001). 


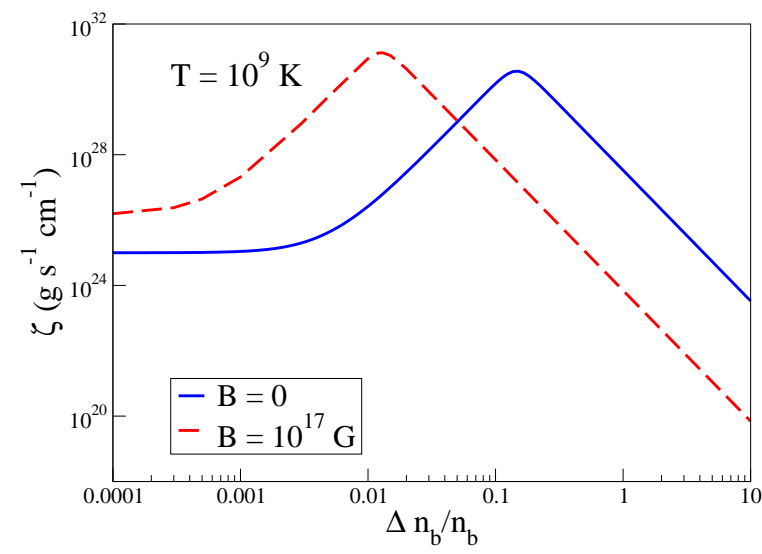

Figure 4: Suprathermal bulk viscosity for $B=0$ and $B=10^{17} \mathrm{G}$ at $T=10^{9} \mathrm{~K}$

[3] P.B. Jones, Phys. Rev. D 64, 084003 (2001).

[4] L. Lindblom and B.J. Owen, Phys. Rev. D 65, 063006 (2002).

[5] W. C. G. Ho and D. Lai, Astrophys. J. 543386 (2000).

[6] J. Madsen, Phys. Rev. D 46, 3290 (1992).

[7] A. Reisenegger and A. A. Bonacic, [astro-ph/0303454].

[8] M. G. Alford, S. Mahmoodifar and K. Schwenzer, J. Phys. G 37 (2010) 125202.

[9] L. B. Leinson and A. Perez, [astro-ph/9711216v2].

[10] P. Haensel, K. P. Levenfish and D. G. Yakovlev, Astron. Astrophys. 357 (2000) 1157.

[11] J. Schaffner and I. N. Mishustin, Phys. Rev. C 531416 (1996).

[12] J. Boguta and A.R. Bodmer, Nucl. Phys. A292, 413 (1977).

[13] B.D. Serot and J.D. Walecka, Adv. in Nucl. Phys. 16, 1 (1986).

[14] M. Sinha and D. Bandyopadhyay, Phys. Rev. D 79, 123001 (2009)

[15] D. A. Baiko and D. G. Yakovlev, Astron. Astrophys. 342192 (1999).

[16] N.K. Glendenning and S.A. Moszkowski, Phys. Rev. Lett. 67, 2414 (1991).

[17] C. B. Dover and A Gal, Prog. Part. Nucl. Phys. 12171 (1984). 